\title{
How does antiviral therapy for hepatitis B influence liver stiffness?
}

\author{
Riccardo Nevola ${ }^{1}$, Luigi E Adinolfi ${ }^{1}$ \& Luca Rinaldi*,1 \\ ${ }^{1}$ Department of Medical, Surgical, Neurological, Metabolic \& Aging Science, University of Campania, Luigi Vanvitelli, Piazza \\ Miraglia, 80138, Naples, Italy \\ *Author for correspondence: Tel.: +39081566 5081; luca.rinaldi@unicampania.it
}

\section{"The changes of LS and its interpretation following antiviral therapies represent a controversial topic."}

First draft submitted: 28 April 2018; Accepted for publication: 9 May 2018; Published online: 20 June 2018

Keywords $\bullet$ antiviral therapy $\bullet$ chronic hepatitis B $\bullet$ fibroscan $\bullet$ liver stiffness $\bullet$ transient elastography

Liver stiffness (LS) is a physical parameter which outlines the elasticity of liver tissue. Fibroscan ${ }^{\circledR}$ (Echosens, Paris, France) is a newly introduced diagnostic instrumentation capable of measuring LS. The transient elastography (TE) method is based on sending an elastic wave through a dedicated probe, provided by a vibrator, placed in the intercostal space at the hepatic right lobe. LS is evaluated according to the speed of the elastic wave in the channel of the hepatic parenchyma and expressed in kilopascal [1]. Several studies have compared the degree of histological fibrosis obtained from liver biopsy and TE. The obtained results showed LS as a reliable marker of liver fibrosis, as well as useful both in liver disease diagnosis and management [2,3]. These findings have allowed an increasingly widespread use of TE in clinical practice, especially in viral hepatitis. TE is a reproducible test, but its reliability is associated with the operator's experience. A technically correct test must contain ten valid measurements, with an interquartile range $<30 \%$, with an increase of reliability for values $<20 \%$ [4]. In addition, the angle between the probe and the hepatic plane must be as perpendicular as possible to allow for a precise evaluation. However, it is also necessary to take into account the possible variability of LS in the portions of liver on which the elastic waves act. In fact, it is already known that the subcapsular regions or the pericicatrizial sites have a higher stiffness than the center-parenchymal portions [5]. These considerations of technical nature establish the possibility, in case of inexperience, to make ten valid measurements with a good interquartile range, but providing a false LS estimate.

Chronic hepatitis $\mathrm{B}(\mathrm{CHB})$ is a disease characterized by a variable pattern, with phases of quiescence and flared necroinflammation [6]. A therapy with nucleos(t)ide analogs, according to international guidelines [7], allows for a sustained virological response and the shutdown of cytolytic activity. In addition, sustained virological response reduced the onset of complications, including hepatocellular carcinoma (HCC) in patients with cirrhosis [8]. Knowledge of the degree of fibrosis at diagnosis represents valuable information for hepatologists in the patient's management. TE has also been shown as an accurate technique in $\mathrm{CHB}$, particularly for the identification of severe fibrosis and cirrhosis [9]. The optimal cut-offs for CHB obtained by Marcellin's study [10] were the following: 7.2 and $11.0 \mathrm{kPa}$ for $\mathrm{F} \geq 2$ and $\mathrm{F}=4$, respectively. The area under receiver-operating characteristic curves were 0.81 (95\% CI: 0.73-0.86) for F $\geq 2,0.93$ (0.88-0.96) for $F \geq 3$ and 0.93 (95\% CI: 0.82-0.98) for F = 4. Subsequently, the meta-analysis of Chon et al. [11] confirmed a good diagnostic accuracy of TE in CHB. In particular, a total of 18 studies on 2772 patients were analyzed. The mean area under receiver-operating characteristic curves for the diagnosis of significant fibrosis (F2), severe fibrosis (F3) and cirrhosis (F4) were 0.859 (95\% CI: 0.857-0.860), 0.887 (95\% CI: 0.886-0.887) and 0.929 (95\% CI: 0.928-0.929), respectively. The estimated cut-off for F2 was 7.9 (range: $6.1-11.8) \mathrm{kPa}$, with a sensitivity of $74.3 \%$ and specificity of $78.3 \%$. For F3, the cut-off value was determined to be 8.8 (range: $8.1-9.7$ ) $\mathrm{kPa}$, with a sensitivity of $74.0 \%$ and specificity of $63.8 \%$; while in the case of F 4 the cut-off was 11.7 (range: 7.3-17.5) kPa, with a sensitivity of $84.6 \%$ and specificity of $81.5 \%$.

The changes of LS and its interpretation following antiviral therapies represent a controversial topic. In our recent study [12], we evaluated LS in a cohort of chronic hepatitis B virus (HBV) patients. In particular, we compared LS 
before antiviral treatment and after a 24-month follow-up. We also compared the subgroup of treated patients with a subset of untreated patients. In the first group we found a median LS decrease of $6 \mathrm{Kpa}$ in patients with advanced fibrosis ( $>8.1 \mathrm{Kpa}$ ) and 1.5 in patients with LS $<8.1 \mathrm{Kpa}$. Conversely, we did not observe significant changes in LS in the untreated patients group. The univariate analysis identified, among factors related to the significant modification of LS, alanine aminotransferase. However, in the multivariate analysis, this parameter did not reach statistical significance. These data suggest that inflammation contributes to the decrease of LS, though not being an independent determining factor. Other studies have assessed the role of cytolysis indices in the decrease of LS with nonunique results [13-17].

Based on our experience we believe, as indicated by Castera's review [18], that a first and rapid reduction of LS is mainly attributable to the disappearance of inflammation. It is only in a second phase, in the subsequent years, that a more gradual reduction of LS may be compatible with an improvement in fibrosis. The cut-offs have been tested in patients with active chronic hepatitis. Due to this reason LS describes the whole fibrosis and necroinflammatory activity and, where present, portal hypertension. This consideration implies that the evaluation of patients with no inflammation and reduced portal pressure may modify LS, regardless of the fibrosis degree. For this reason, the reference cut-off for moderate fibrosis and cirrhosis with a good accuracy in patients with active infection, cannot guarantee the same reliability in the long-term responder (LTR) patients.

The improvement of fibrosis or the regression of cirrhosis is the main target in LTR patients, but this success could be only partially possible [19]. Marcellin et al. [20] carried out a study on a large cohort of CHB patients, performing liver biopsies prior to therapy and then after 5 years of monitoring in LTR patients. The results showed that the $74 \%$ of patients with cirrhosis had a reduction in fibrosis at year 5 and were no longer cirrhotic. More than the half $(58 \%)$ of this subgroup had a decrease of three units or more in the Ishak score at 5 years.

In conclusion, we believe that Fibroscan is a reliable tool in the management of $\mathrm{CHB}$ patients. The early changes of LS after antiviral treatment is mainly enabled by the effect of viral clearance and shutdown of inflammation; while the further and gradual reduction may be due to an improvement in fibrosis, but this cannot be proven without histological data.

\section{Acknowledgements}

PC Pafundi, for the English grammatical and style revision; MC Fascione, N luliano and A Amelia for editorial assistance.

\section{Financial \& competing interests disclosure}

The authors have no relevant affiliations or financial involvement with any organization or entity with a financial interest in or financial conflict with the subject matter or materials discussed in the manuscript. This includes employment, consultancies, honoraria, stock ownership or options, expert testimony, grants or patents received or pending, or royalties.

No writing assistance was utilized in theproduction of this manuscript.

\section{References}

1. Rinaldi L, Valente G, Piai G. Serial liver stiffness measurements and monitoring of liver-transplanted patients in a real-life clinical practice. Hepat. Mon. 16(12), e41162 (2016).

2. Vitrone M, Iossa D, Rinaldi L et al. Hepatitis B virus reactivation after heart transplant: incidence and clinical impact. J. Clin. Virol. 96, 54-59 (2017).

3. Lee HW, Yoo EJ, Kim BK et al. Prediction of development of liver-related events by transient elastography in hepatitis B patients with complete virological response on antiviral therapy. Am. J. Gastroenterol. 109(8), 1241-1249 (2014).

4. Boursier J, Zarski JP, de Ledinghen V et al. Multicentric group from ANRS/HC/EP23 FIBROSTAR studies. Determination of reliability criteria for liver stiffness evaluation by transient elastography. Hepatology 57(3), 1182-1191 (2013).

5. Maharaj B, Maharaj RJ, Leary WP et al. Sampling variability and the influence on the diagnostic yield of percutaneous needle biopsy of the liver. Lancet 1(8480), 523-525 (1986).

6. Marrone A, Capoluongo N, D'Amore C et al. Eighteen-month-lamivudine-prophylaxis on preventing occult hepatitis B virus (HBV) infection reactivation in patients with hematologic malignancies receiving immunosuppression therapy. J. Viral. Hepat. 25(2), 198-204 (2018).

7. European Association For The Study of the Liver. EASL 2017 clinical practice guidelines on the management of hepatitis B virus infection. J. Hepatol. 67(2), 370-398 (2017).

8. Lok AS, Zoulim F, Dusheiko G, Ghany MG. Hepatitis B cure: from discovery to regulatory approval. Hepatology 66(4), 1296-1313 (2017). 
9. Branchi F, Conti CB, Baccarin A, Lampertico P, Conte D, Fraquelli M. Non-invasive assessment of liver fibrosis in chronic hepatitis B. World J. Gastroenterol. 20(40), 14568-14580 (2014).

10. Marcellin P, Ziol M, Bedossa P et al. Non-invasive assessment of liver fibrosis by stiffness measurement in patients with chronic hepatitis B. Liver Int. 29(2), 242-247 (2009).

11. Chon YE, Choi EH, Song KJ et al. Performance of transient elastography for the staging of liver fibrosis in patients with chronic hepatitis B: a meta-analysis. PLoS ONE 7(9), e44930 (2012).

12. Rinaldi L, Ascione $\mathrm{A}, \mathrm{Messina} \mathrm{V}$ et al. Influence of antiviral therapy on the liver stiffness in chronic HBV hepatitis. Infection 46(2), 231-238 (2018).

13. Kim MN, Kim SU, Kim BK et al. Long-term changes of liver stiffness values assessed using transient elastography in patients with chronic hepatitis B receiving entecavir. Liver. Int. 34(8), 1216-1223 (2014).

14. Kuo YH, Lu SN, Chen $\mathrm{CH}$ et al. The changes of liver stiffness and its associated factors for chronic hepatitis B patients with entecavir therapy. PLoS ONE 9(3), e93160 (2014).

15. Christiansen KM, Mössner BK, Hansen JF et al. Liver stiffness measurement among patients with chronic hepatitis B and C: results from a 5-year prospective study. PLoS ONE 9(11), e111912 (2014).

16. Jang W, Yu SI, Sinn DH et al. Longitudinal change of liver stiffness by transient elestography in chronic hepatitis B patients treated with nucleos(t)ide analogue. Clin. Res. Hepatol. Gastroenterol. 38(2), 195-200 (2014).

17. Kim JH, Kim MN, Han KH, Kim S. Clinical application of transient elastography in patients with chronic viral hepatitis receiving antiviral treatment. Liver Int. 35(4), 1103-1115 (2015).

18. Castera L. Hepatitis B: are non-invasive markers of liver fibrosis reliable? Liver Int. 34(Suppl. 1), 91-96 (2014).

19. Bedossa P. Reversibility of hepatitis B cirrhosis after therapy: who and why? Liver Int. 35(Suppl. 1), 78-81 (2014).

20. Marcellin P, Gane E, Buti M et al. Regression of cirrhosis during treatment with tenofovir disoproxil fumarate for chronic hepatitis B: a 5-year open-label follow up study. Lancet 381(9865), 468-475 (2013). 
